\title{
Extraction and Antioxidant Activity Evaluation of Polyphenols of Brown Rice Tea from Japonica Rice and Indica Rice
}

\author{
Shuangqi Tian ${ }^{1}$, ZhichengChen ${ }^{2 *}$,Kejing Yang ${ }^{3}$ \\ ${ }^{1,2,3}$ College of Food Science and Technology, Henan University of Technology, Zhengzhou 450001, China
}

Received: 12 February, 2020; Accepted: 12 March, 2020; Published: 16 March, 2020

*Corresponding author: ZhichengChen,College of Food Science and Technology, Henan University of Technology, Zhengzhou 450001, China. Email: chen_1958@163.com

\begin{abstract}
The objective of this study was to explore the extraction process and antioxidant activity of polyphenols from Japonica and Indica brown rice. After baking, Japonica and Indica brown rice samples were soaked in water to obtain brown rice tea respectively. We determined the polyphenol content and the antioxidant activity of the brown rice tea, as well as measured the contents of nutrients (including protein, amino acid, and crude cellulose) of the baked brown rice. The total AA content of Indica brown rice (7.30\%) was higher than that of Japonica brown rice (6.30\%). The results showed that the optimum extraction conditions for Japonica and Indica rice polyphenols were as follows: baking temperature of $180^{\circ} \mathrm{C}$ and $190^{\circ} \mathrm{C}$, baking time of $15 \mathrm{~min}$ and $20 \mathrm{~min}$, and soaking time of $30 \mathrm{~min}$ and $30 \mathrm{~min}$, respectively. The DPPH clearance rate of the five samples was shown as follows: Burdock tea> Pu'er tea> Tieguanyin tea> Japonica brown rice> Indica brown rice tea. The DPPH clearance rate of the polyphenols in rice tea obtained from Japonica and Indica brown rice was 1.04\% and 1.99\%.
\end{abstract}

Keywords : Japonica rice; Indica rice; Polyphenols; Brown rice tea

\section{Introduction}

Obesity is significantly associated with the development of some diseases, including type 2 diabetes, heart disease, insulinoma, cardiac disease, hypertension, polycystic ovary syndrome, osteoarthritis and lipid metabolism disorder [1]. A healthier lifestyle choice is not always effective against obesity. Therefore, to suppress the epidemic of obesity, therapeutic treatment is required, however, medical treatment for obesity is limited. Due to the adverse side effects of safety concerns, most drug treatments have not been successful, there is a high demand for alternative treatment options [2]. Incorporating functional foods with bioactive properties into the diet can be a potential supplement to obesity treatment.

Rice (Oryza Sativa L.) is a staple food that is consumed by at least half of the world's population at least once a day and is planted in more than 100 countries [3]. In particular, colored rice varieties have been shown to have anti-inflammatory and antioxidant properties thus making rice as a potential candidate for nutritional supplements and functional food substitutes. Bran of whole rice varieties has shown therapeutic properties due to the presence of polyphenols $[4,5]$.

Polyphenols belong to a subclass of phytochemicals and dominate in grains, fruits and vegetables [6]. The incorporation of polyphenols into the diet through rice consumption may be a potential solution to reduce the incidence of metabolic syndrome and its associated risk factors such as obesity. Therefore, the rice breeding program is based on the development of rice varieties that not only appeal to consumers' taste and texture characteristics, but also are rich in polyphenols with potential antioxidant and anti-inflammatory properties [7]. It is important to satisfy health-conscious consumers. The brown rice can be used as functional foods to satisfy their cooking and nutritional needs. Brown rice has potentials in resisting oxidation and antioxidants, diminishing inflammation and reducing blood lipids. This potential of rice-derived polyphenols may potentially regulate risk factors for obesity-related rice and inflammation development [8].

Rice is composed of different layers, and the outer layer is generally called the hull, which accounts for $16 \%$ to $28 \%$ the weight of rice [9]. In a process known as shelling, the hull is removed to expose the bran. The resulting product is commonly referred to as whole grain rice. Whole grain rice is the endosperm (white rice) and the bran layer is still intact [10]. Bran consists of several layers (peel, seed coat, bead and aleurone), accounting for $6 \%-7 \%$ the total weight of rice. Most of the polyphenols are present in these ectopic layers of the endosperm [9]. Therefore, the main objective of this research was to investigate the content and the antioxidant activity of polyphenols in baked brown rice tea.

\section{Materials and Methods}

\section{Materials}

Japonica rice was obtained from Daxie Yushu Township Panjin Daqingshan Rice Processing Factory (Liaoning, China). Indica rice was purchased from Nankou Town, Meixian District, Meizhou (Guangdong, China). Tieguanyin was purchased from Longxi Township, Anxi County, (Fujian, China), and Pu'er Tea was 
purchased from Yunnan Puqi Kang Tea Co., Ltd. (Yunnan, China). Burdock tea was purchased from China's burdock village, Lanling (Shandong, China). All samples were subjected to the same post-harvest handling and storage at $4^{\circ} \mathrm{C}$. Analysis of all the biological replicates was conducted in triplicate. The chemicals were of analytical reagent grade.

\section{Preparation of Brown Rice Tea and Control Teas}

The brown rice was placed in a constant temperature oven from Nantong Huatai Experimental Instrument Co., Ltd. (Jiangsu, China) for drying. About $10 \mathrm{~g}$ of brown rice was weighed by an electronic balance using a $100 \mathrm{~mL}$ beaker, baked at 180 ? for $15 \mathrm{~min}$, then soaked with $60 \mathrm{~mL}$ boiling water for $20 \mathrm{~min}$. Control teas were soaked in boiling water for $20 \mathrm{~min}$. As shown in Figure 1, the preparation process of brown rice tea mainly included baking, and socking.

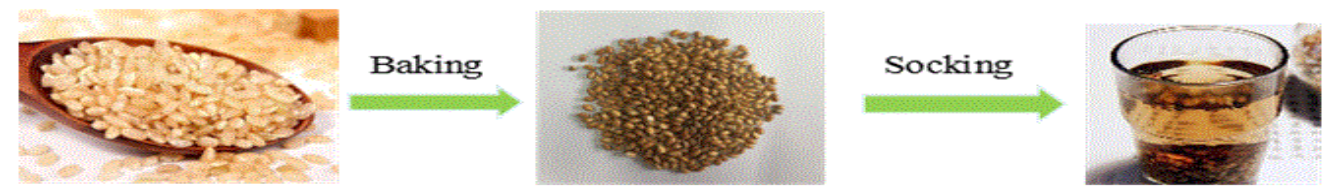

Figure 1: Experimental Design

\section{Nutrients Analysis of the Brown Rice}

The protein content was determined by the Kjeldahl method. A $0.5 \mathrm{~g}$ sample was mixed with $4 \mathrm{~mL}$ concentrated sulfuric acid in a $100 \mathrm{~mL}$ round bottom flask, and the mixture was heated to 440? using a conventional convection conductive heating system until boiling. However, the heating time did not exceed 3-5 min. The crude cellulose content was estimated by the method of Ahuja and Bajaj and the value was expressed as a percentage of cellulose equivalent. Amino acid analysis of rice was carried out according to the method of $\mathrm{Du}[11,12]$. Rice flour $(100 \mathrm{mg})$ was hydrolyzed with $10 \mathrm{~mL} 5 \mathrm{~mol} / \mathrm{L} \mathrm{NaOH}$ at 110 目 for $20 \mathrm{~h}$. The mixture was transferred and dissolved in deionized water in a 50 $\mathrm{mL}$ volumetric flask. However, the solution was filtered through a $0.45 \mu \mathrm{m}$ nylon syringe filter (Filtrex Technology, Singapore). The amount of each Amino Acid (AA) was determined using an automatic amino acid analyzer (Biochrom 30+, Cambridge, UK). Amino acids were post-column derivatized with ninhydrin reagent $(0-50 \mathrm{~mL} / \mathrm{h})$ and detected by absorbance at $570 \mathrm{~nm}$ and $440 \mathrm{~nm}$. Amino acids and standard solutions were analyzed under the same conditions.

\section{Determination of Total Phenolic Content (TPC)}

The total free phenol content was determined using the method described by Qiu et al.[13]. Briefly, a $1 \mathrm{~mL}$ sample was incubated with $5 \mathrm{~mL}$ Folin-Ciocalteu reagent for $5 \mathrm{~min}$ in the dark. The mixture was neutralized by the addition of $4 \mathrm{~mL} 7 \%$ sodium carbonate solution and $1 \mathrm{~mL}$ of deionized water. After incubation for $90 \mathrm{~min}$ in the dark, the absorbance was measured at $765 \mathrm{~nm}$ against a methanol blank on a microplate reader (BMG Labtech FLUOstar Omega, Offenburg, Germany). The total phenolic content of the rice samples was expressed as $\mathrm{mg} / 100 \mathrm{~g}$ gallic acid equivalent (GAE).

\section{Antioxidant Activity Determination}

The free radical scavenging activity was determined by 2,2-diphenyl -picrylhydrazyl (DPPH) assay. The value of DPPH represents the antioxidant capacity of a given substance compared to standard Trolox. This method is based on the decolorization of the stable free radical DPPH. When DPPH is mixed with a solution of the substance that can provide hydrogen atoms, the result produces a reduced form while losing purple to yellow color [14]. Spectrophotometric analysis was performed as published by Brand-Williams et al. [15]. To determine free radical scavenging activity, $1.45 \mathrm{~mL}$ DPPH colored groups were added to $50 \mathrm{~mL}$ diluted sample extract or Trolox (standard) in methanol. The mixture was allowed to stand in the dark at room temperature for $30 \mathrm{~min}$. The absorbance was measured at 515 $\mathrm{nm}$ [16]. Use $0.05 \mathrm{mmol} / \mathrm{L}(0.0125 \mathrm{mg} / \mathrm{mL})$ to $1 \mathrm{mmol} / \mathrm{L}(0.25$ $\mathrm{mg} / \mathrm{mL}$ ) Trolox (6-hydroxy -2,5,7,8-tetramethyl -chroman-2carboxylic acid) methanol, the solution is calibrated. The free radical scavenging activity was expressed as Trolox equivalent $g$ per $100 \mathrm{~g}$ dry matter. DPPH, methanol and Trolox were purchased from French VWR.

\section{Statistical Analysis}

All experimental data were estimated in triplicate and all statistical calculations were performed using statistical analysis software Origin Lab 9.0 (Origin Lab Corporation). Significant differences $(\mathrm{P} \leq 0.05)$ among various treatments were detected by Duncan's multiple range tests.

\section{Results and Discussion}

\section{Nutritional Characteristics of Japonica Brown Rice and Indica Brown Rice}

As shown in Table 1, the protein content $(8.08 \%)$ and crude cellulose content (1.29\%) of Indica brown rice were higher than those of Japonica brown rice $(7.72 \%$ and $1.05 \%)$. The total AA content of Indica brown rice $(7.30 \%)$ was higher than that of Japonica brown rice (6.30\%). The content of Glu, Asp, Thr, Ser, and Tyr in Indica brown rice was higher than that in Japonica brown rice, respectively. In particular, the content of Thr was increased by $50 \%$. These ensured the nutritional value of brown rice tea. 


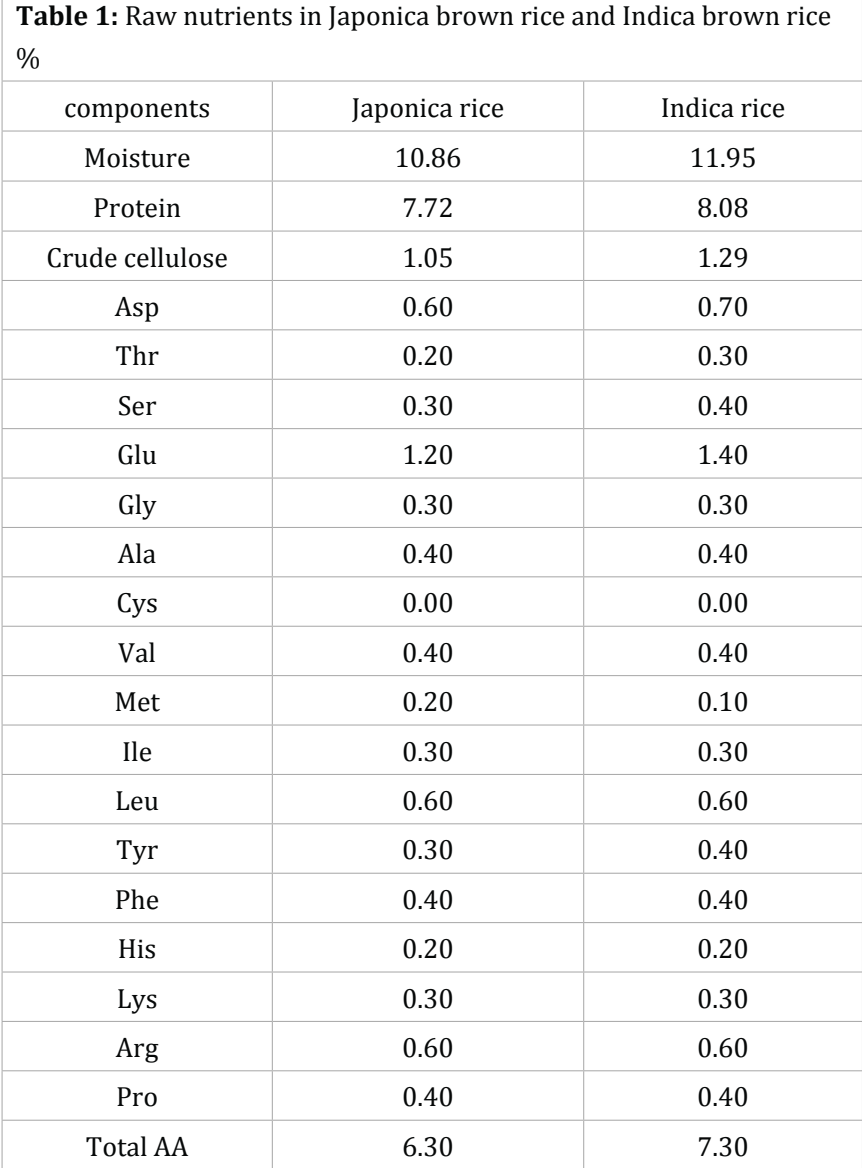

Effects of Different Factors on Polyphenols Content in Japonica Brown Rice Tea

As shown in Figure 2A, under the conditions of constant baking time and soaking time, the polyphenols content in the Japonica brown rice tea rose first and then fell with the increase of baking temperature. The polyphenols content reached the highest $(49.69 \mu \mathrm{g} / \mathrm{mL})$ as the baking temperature was $180^{\circ} \mathrm{C}$. As shown in Figure $2 \mathrm{~B}$, under the conditions of constant baking temperature and soaking time, the polyphenols content in the brown rice tea rose first and then fell with the extension of baking time, and the polyphenols content $(49.62 \mu \mathrm{g} / \mathrm{mL})$ reached the highest as baking for 20 min. As shown in Figure 2C, under the conditions of constant baking temperature and baking time, the polyphenols content in the brown rice tea rose first and then fell with the immersion time, and the polyphenols content $(49.78 \mu \mathrm{g} / \mathrm{mL})$ reached the highest as immersing for $25 \mathrm{~min}$.

For Japonica brown rice, it can be seen from Figure 2A-2C that the polyphenols content in tea rose first and then fell with the increase of baking temperature, baking time and soaking time. For the baking temperature, select $170,180,190{ }^{\circ} \mathrm{C}$ for three levels; $180^{\circ} \mathrm{C}$ for the optimum baking temperature, soaking time was still $20 \mathrm{~min}$, for baking time, 10 and $25 \mathrm{~min}$. The polyphenols content was not different. Considering baking for $10 \mathrm{~min}$, the time was a little short, some brown rice was not cooked enough,
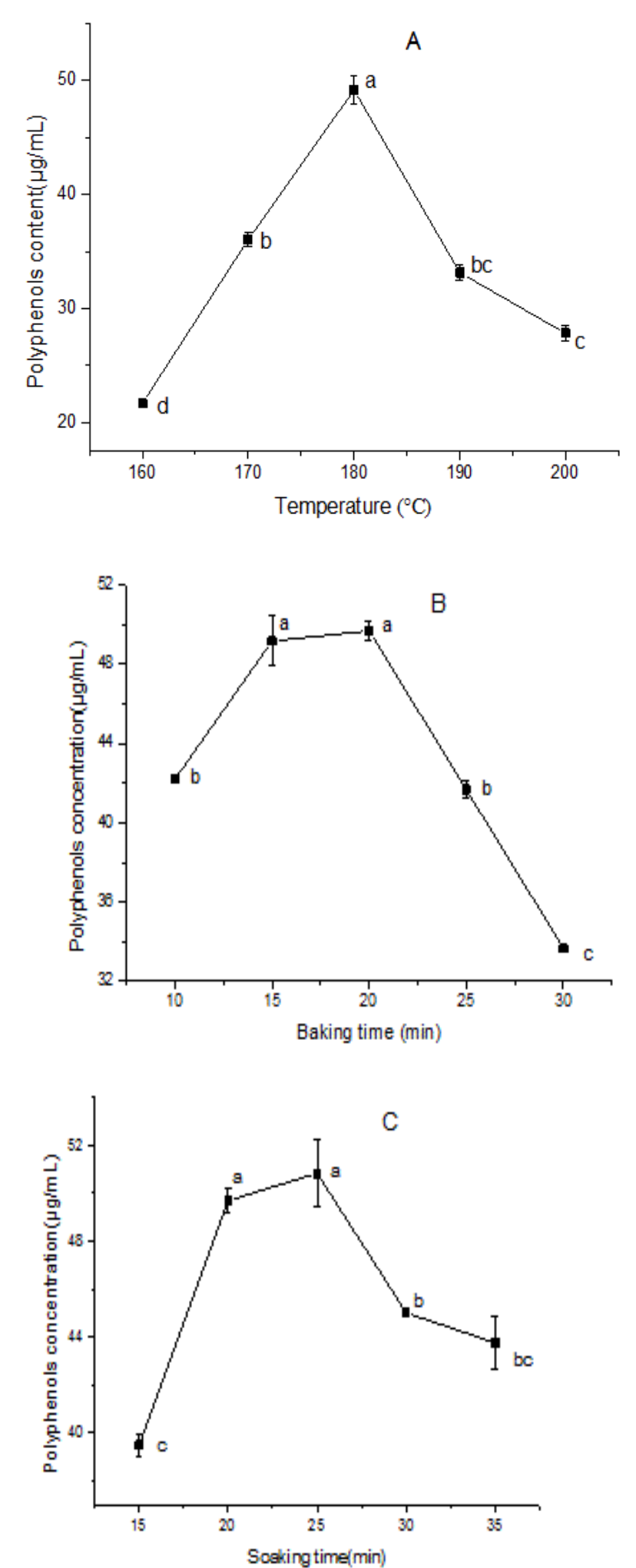

Figure 2: Effects of different factors on polyphenols content in Japonica brown rice tea. a-dMean values with different letters are significantly different $(p \leq 0.05)$ at the same treatment conditions 
so choose $15,20,25 \mathrm{~min}$ for three levels; $180^{\circ} \mathrm{C}$ was the optimum baking temperature, $20 \mathrm{~min}$ was the optimum baking time. For the soaking time, the tea temperature was not cool at $30 \mathrm{~min}$, and the tea temperature was not hot at $20 \mathrm{~min}$, which was suitable for drinking tea. The selection was 20,25, 30 min for three levels. As shown in Table 2, the polyphenols content in Japonica brown rice tea under optimal conditions by orthogonal experiment was the baking temperature is $180^{\circ} \mathrm{C}$, the baking time is $15 \mathrm{~min}$, and the soaking time was $30 \mathrm{~min}$. Baking time is the most important factor in all treatment conditions.

Table 2: Orthogonal experiment of polyphenols content in Japonica brown rice tea

\begin{tabular}{|c|c|c|c|c|}
\hline Treatments & A & B & $\mathrm{C}$ & \multirow{2}{*}{$\begin{array}{c}\text { Polyphenols } \\
\text { content }(\mu \mathrm{g} / \\
\mathrm{mL})\end{array}$} \\
\hline & $\begin{array}{c}\text { Temperature } \\
\left({ }^{\circ} \mathrm{C}\right)\end{array}$ & $\begin{array}{l}\text { Baking } \\
\text { time } \\
\text { (min) }\end{array}$ & $\begin{array}{l}\text { Soaking } \\
\text { time(min) }\end{array}$ & \\
\hline 1 & $1(170)$ & $1(15)$ & $1(20)$ & $46.16 \pm 1.21$ \\
\hline 2 & 1 & $2(20)$ & $2(25)$ & $43.03 \pm 1.02$ \\
\hline 3 & 1 & $3(25)$ & $3(30)$ & $46.78 \pm 0.96$ \\
\hline 4 & $2(180)$ & 1 & 2 & $53.45 \pm 1.37$ \\
\hline 5 & 2 & 2 & 3 & $39.28 \pm 1.03$ \\
\hline 6 & 2 & 3 & 1 & $43.76 \pm 1.16$ \\
\hline 7 & $3(190)$ & 1 & 3 & $48.55 \pm 0.81$ \\
\hline 8 & 3 & 2 & 1 & $44.49 \pm 1.19$ \\
\hline 9 & 3 & 3 & 2 & $35.74 \pm 0.57$ \\
\hline $\begin{array}{l}\text { Laying } \\
\text { style } 1\end{array}$ & 0.226 & 0.239 & 0.224 & \\
\hline $\begin{array}{l}\text { Laying } \\
\text { style } 2\end{array}$ & 0.227 & 0.217 & 0.222 & \\
\hline $\begin{array}{l}\text { Laying } \\
\text { style } 3\end{array}$ & 0.219 & 0.216 & 0.225 & \\
\hline $\begin{array}{c}\text { Measures of } \\
\text { variation }\end{array}$ & 0.008 & 0.023 & 0.003 & \\
\hline
\end{tabular}

\section{Effects of Different Factors on Polyphenols Content in Indica} Brown Rice Tea

As shown in Figure 3A, under the conditions of constant baking time and soaking time, the polyphenols content in the Indica brown rice tea rose first and then fell with the increase of baking temperature, and the polyphenols content $(44.93 \mu \mathrm{g} /$ $\mathrm{mL}$ ) reached the highest as the drying temperature was $180^{\circ} \mathrm{C}$. As shown in Figure 3B, under the conditions of constant baking temperature and soaking time, the polyphenols content in the brown rice tea rose first and then fell with the extension of baking time, and the polyphenols content $(49.15 \mu \mathrm{g} / \mathrm{mL})$ reached the highest as baking for $20 \mathrm{~min}$. As shown in Figure 3C, under the conditions of constant baking temperature and baking time, the
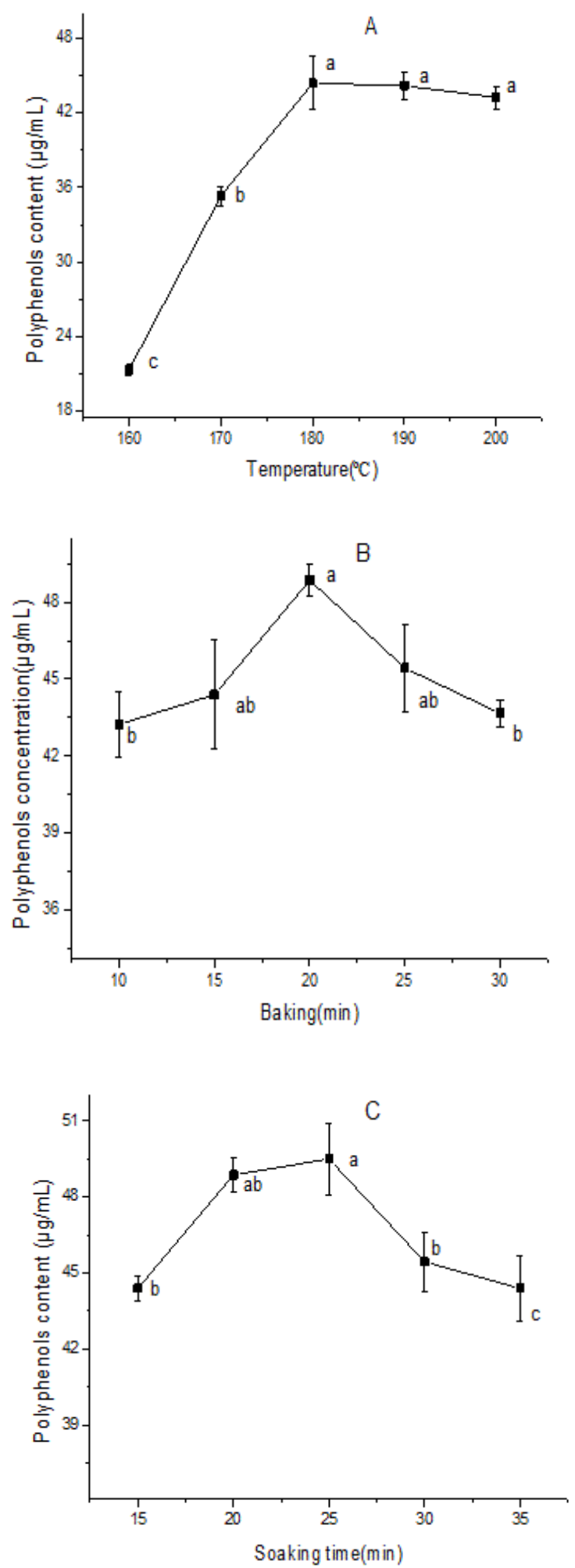

Figure 3: Effects of different factors on polyphenols content in Indica brown rice tea. a-dMean values with different letters are significantly different $(\mathrm{p} \leq 0.05)$ at the same treatment conditions 
polyphenols content in the brown rice tea rose first and then fell with the immersion time, and the polyphenols content $(49.46 \mu \mathrm{g} /$ $\mathrm{mL}$ ) reached the highest when immersing for $25 \mathrm{~min}$.

For the brown rice, it can be seen from Figure 3A-3C that the polyphenols content in the tea rose first and then fell with the increase of baking temperature, baking time and soaking time. For the baking temperature, although the polyphenols content was high at $200{ }^{\circ} \mathrm{C}$, since the brown rice has been burnt at this temperature, three levels of 170,180 , and $190^{\circ} \mathrm{C}$ were selected; $180^{\circ} \mathrm{C}$ was the optimum baking temperature. For the baking time, 15, 20 and 25 min were selected as three levels. For the soaking time, the temperature of tea was not cool at $30 \mathrm{~min}$, and the temperature of tea was not hot at $20 \mathrm{~min}$. It was suitable for drinking tea. It was selected from three levels of 20,25 and $30 \mathrm{~min}$. As shown in Table 3, the polyphenols content in Japonica brown rice tea under optimal conditions by orthogonal experiment was the baking time is $190^{\circ} \mathrm{C}$, the baking time was $20 \mathrm{~min}$, and the soaking time is $30 \mathrm{~min}$. Baking time is the most important factor in all treatment conditions.

\section{Antioxidant Activity of Five Different Teas}

As shown in Figure 4, about the determination of the antioxidant activity of five different teas, the tea concentration of Japonica brown rice and Indica brown rice was $0.017 \mathrm{~g} / \mathrm{mL}$, and the tea concentration of Tieguanyin, Pu'er and Burdock was $0.83 \times 10-3$ $\mathrm{g} / \mathrm{mL}$. The DPPH clearance rate of the five samples was shown as follows: Burdock tea $>$ Pu'er tea $>$ Tieguanyin tea $>$ Japonica brown rice> Indica brown rice tea. However, the DPPH clearance rate of the polyphenols in rice tea obtained from Japonica and Indica brown rice was $1.04 \%$ and $1.99 \%$.

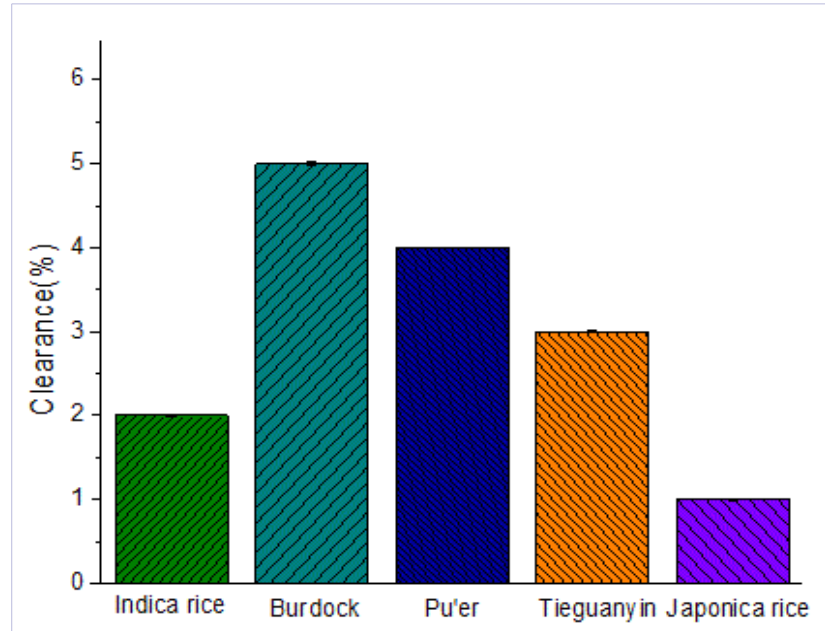

Figure 4: Antioxidant activity of the obtained extracts of different teas

\section{Conclusions}

Most of the polyphenols are present in the ectopic layers of the endosperm from Japonica rice and Indica rice. Therefore, the main objective of this research was to investigate the polyphenol content of baking brown rice and the effect of polyphenols on antioxidant activity. Soaking for tea, the remaining Japonica brown rice and Indica brown rice were boiled into rice, and then antioxidant activity evaluation of the brown rice tea was carried out. Then, the nutrients of Japonica brown rice and Indica brown rice, including moisture content, protein content, amino acid content, and crude cellulose content were determined. The total AA content of Indica brown rice (7.30\%) was higher than that of Japonica brown rice $(6.30 \%)$. Finally, several kinds of common tea were selected for comparative study. The polyphenols in tea obtained from Japonica and Indica brown rice were shown as potential DPPH clearance rates. The DPPH clearance rate of the polyphenols in rice tea obtained from Japonica and Indica brown rice was $1.04 \%$ and $1.99 \%$.

\section{Acknowledgment}

The authors would like to acknowledge NSFC for financial assistance under NSFC Research Contract no. 31701636, and the Science and Technology Research Project of the department of science and technology of Henan Province (NO: 182102110394).

\section{References}

1. DPGuh, WZhang, N Bansback, Z Amarsi, CL Birmingham, AH Anis. The incidence of co-morbidities related to obesity and overweight: a systematic review and meta-analysis. BMC Public Health. 2009;9:88. doi: 10.1186/1471-2458-9-88

2. AK Kakkar, N Dahiya. Drug treatment of obesity: current status and future prospects. Eur J Intern Med. 2015;26(2):89-94.

3. SMohantay. Trends in global rice consumption, Rice Today. 12(2013):44-45.

4. M Candiracci, ML Justo, A Castaño, R Rodriguez-Rodriguez, MD Herrera. Antioxidant effects of anthocyanins-rich extract from black sticky rice on human erythrocytes and mononuclear leukocytes. Afr J Biotechnol. 2010;9(48):466-472.

5. Manila Candiracci, Maria Luisa Justo, Angelica Castaño, Rosalia Rodriguez-Rodriguez, Maria Dolores Herrera. Rice bran enzymatic extract-supplemented diets modulate adipose tissue inflammation markers in Zucker rats. Nutrition. 2014;30(4):466-472.

6. B Santhakumar, AC Bulmer, I Singh. A review of the mechanisms and effectiveness of dietary polyphenols in reducing oxidative stress and thrombotic risk. J Hum Nutr Diet. 2014;27(1):1-21.

7. SW Min, SN Ryu, DH Kim. Anti-inflammatory effects of black rice, cyanidin-3-0- $\beta$-D-glycoside, and its metabolites, cyanidin and protocatechuic acid. Int Immunopharmacolo. 2010;10(8):959-966.

8. ET Callcott, AB Santhakumar, JLuo, CL Blanchard. Therapeutic potential of rice-derived polyphenols on obesity-related oxidative stress and inflammation, J Appl Biomed. 2018;16(4):255-262.

9. Gunaratne, K Wu, DLi, A Bentota, H Corke, YZ Cai. Antioxidant activity and nutritional quality of traditional red-grained rice varieties containing proanthocyanidins. Food Chem. 2013;138(2-3):11531161.

10. L Kealey, W Clampett. Production of quality rice in south eastern Australia, Weed Management. Kingston, A.C.T. Rirdc, 2000.

11. H Chen, TJSiebenmorgen, KGriffin. Quality characteristics of long-grain rice milled in two commercial systems, Cereal Chem. 
1998;75(4):560-565.

12. KL Ahuja, KL Bajaj. Colorimetric determination of crude fibre in cruciferous oilseeds. Cruciferae Newsl. 1999;21:61-62.

13. PDu, X Wang, C Xu, YGao. PseAAC-Builder: A cross-platform standalone program for generating various special Chou's pseudo-amino acid compositions. Anal Biochem. 2012;425(2):117-119.doi: 10.1016/j.ab.2012.03.015

14. YQiu, QLiu, TBeta. Antioxidant properties of commercial wild rice and analysis of soluble and insoluble phenolic acids. Food Chem. 2010;121(1):140-147.
15. P Molyneux. The use of the stable free radical diphenyl picrylhydrazyl(DPPH) for estimating antioxidant activity. Songklanakarin J Sci and Tech. 2003;26(2):211-219.

16.W Brand-Williams, ME Cuvelier, C Berset. Use of a free radical method to evaluate antioxidant activity. LWT Food science and technology. 1995;28(1):25-30.

17. Barba, MJ Esteve, AFrigola. Physicochemical and nutritional characteristics of blueberry juice after high pressure processing. Food Res Int. 2013;50(2):545-549. 